\title{
Análisis de las políticas de enseñanza universitaria en España en tiempos de Covid-19. La presencialidad adaptada
}

\section{Analysis of Higher Education Policies in Spain for Covid-19: Adapted Presence}

\author{
Manuel Area-Moreira \\ Universidad de La Laguna. La Laguna, España \\ manarea@ull.edu.es \\ Anabel Bethencourt-Aguilar \\ Universidad de La Laguna. La Laguna, España \\ abethenc@ull.edu.es \\ Sebastián Martín-Gómez \\ Universidad de La Laguna. La Laguna, España \\ smarting@ull.edu.es \\ $M^{a}$ Belén San Nicolás-Santos \\ Universidad de La Laguna. La Laguna, España \\ bsannico@ull.edu.es
}

\section{Resumen:}

En este artículo se analizan las propuestas de política educativa que las universidades públicas españolas han preparado para el nuevo curso escolar 2020-21 condicionado por la pandemia de la pandemia Covid-19. El estudio se basa en las recomendaciones ofrecidas por el Ministerio de Universidades del Gobierno de España, así como las distintas resoluciones o indicaciones que han realizado los equipos de gobierno de distintas universidades españolas. El estudio pretende dar respuesta a tres cuestiones básicas: ¿En qué consisten las políticas destinadas a la implementación de la presencialidad adaptada? ¿Existe consenso o disonancia en la interpretación de este concepto? ¿Qué visiones pedagógicas y estratégicas subyacen a las políticas de las universidades españolas sobre la "presencialidad adaptada"?

El artículo concluye señalando que existe una política similar para el sistema universitario español derivado de las recomendaciones del Ministerio de Universidades sobre la presencialidad adaptada. Asimismo, se indica que dicho concepto no existe en la bibliografía académica, pero que responde a la modalidad de la enseñanza semipresencial, híbrida o de blended learning. Se finaliza señalando que estas políticas son de corto plazo y que responden a la emergencia sanitaria careciendo de una visión estratégica sobre la transformación digital de la educación superior para los tiempos posCovid19.

Palabras claves: Educación Superior, Covid-19, transformación digital, educación a distancia, enseñanza semipresencial 
RED. Revista de Educación a Distancia. Núm. 65, Vol. 21. Artíc. 3, 08-01-2021.

DOI: https://doi.org/10.6018/red.450461

\begin{abstract}
:
This article analyzes the educational policy proposals that the Spanish public universities have prepared for the new 2020-21 school year conditioned by the Covid-19 pandemic. The study is based on the recommendations offered by the Ministry of Universities of the Government of Spain, as well as the different resolutions or indications that the government teams of different Spanish universities have made. The study tries to answer three basic questions: What are the policies for the implementation of adapted presence? Is there consensus or dissonance in the interpretation of this concept? What strategic visions underlie the policies of Spanish universities on "adapted presence"?

The article concludes by noting that there is a similar policy for the Spanish public university system derived from the recommendations of the Ministry of Universities on adapted face-to-face attendance. It is also indicated that this concept does not exist in the academic bibliography, but that it responds to the modality of blended, hybrid or blended learning teaching. It ends by noting that these policies are short-term and that they respond to the health emergency, lacking a strategic vision on the digital transformation of higher education for the post-Covid-19 times.
\end{abstract}

Keywords: Higher Education, Covid-19, digital transformation, distance education, blended learning

\title{
Introducción
}

Desde la declaración de la pandemia de la Covid-19 en marzo de 2020 se ha generado una importante cantidad de publicaciones con relación al impacto de la misma sobre las instituciones educativas tanto de nivel primario, secundario y superior. Sobre este particular distintos organismos internacionales han realizado recomendaciones específicas sobre cómo abordar la enseñanza en los ámbitos escolares a través de modalidades formativas a distancia a través de la red. La OCDE ha sido pródiga en la publicación de este tipo de guías (OECD and Harvard Graduate School of Education, 2020; Gouëdard, Pont and Viennet, 2020; Reimers y Schleicher, 2020 a; b). La UNESCO también ha publicado sus propios documentos de análisis del impacto de la Covid sobre sistemas escolares (2020a y b) ofreciendo, a partir de los mismos, recomendaciones a los gestores y administraciones gubernamentales para reabrir las escuelas (UNESCO, 2020c).

Desde estos organismos se propone, entre otras medidas, en evitar y/o compensar las brechas de desigualdad en el acceso a la tecnología por parte del alumnado perteneciente a grupos sociales desfavorecidos, en incrementar la formación y competencia digital del profesorado, en articular sistemas de plataformas y recursos digitales para la comunicación online entre el alumnado y los docentes, así como en ofrecer variedad de materiales didácticos digitales abiertos para la cumplimentación de las tareas escolares desde el hogar.

En el ámbito de la educación superior también se han publicado diversos estudios y ensayos que exploraron los efectos más relevantes del cierre de las aulas y espacios

Análisis de las políticas de enseñanza universitaria en España para tiempos Covid19: la presencialidad adaptada. M. Area. A. Bethencourt, S. Martín y B. San Nicolás.

Página 2 de 19 
RED. Revista de Educación a Distancia. Núm. 65, Vol. 21. Artíc. 3, 08-01-2021.

DOI: https://doi.org/10.6018/red.450461

universitarios como consecuencia de la pandemia ${ }^{1}$. Al respecto pueden verse trabajos como el de Crawford y otros (2020) donde describen dicho impacto en las universidades de veinte países. Entre las conclusiones de este trabajo comparado se señala que existen variedad de respuestas. Por un lado, un grupo de universidades hizo muy poco para responder y optó por cumplir con los estándares mínimos de su gobierno manteniendo la enseñanza presencial. Por otro lado, otras universidades cerraron su oferta presencial y pasaron a la educación digitalizada. Otras, ya estaban parcialmente preparadas ya que tenían experiencia en combinar la enseñanza presencial y la educación a distancia en línea.

En esta misma dirección hemos de referirnos al informe elaborado por la International Association of Universities (Marinoni, Land and Jensen, 2020) basado en una encuesta que fue respondida por directivos de 424 universidades y otras instituciones de educación superior con sede en 109 países y dos regiones administrativas de China (Hong Kong y Macao). De la misma también se pone de manifiesto que el tipo de respuesta fue desigual entre las universidades, aunque dos tercios indican que la enseñanza en el aula fue reemplazada por la enseñanza a distancia. El cambio de la enseñanza presencial a la enseñanza a distancia no se produjo sin desafíos y obstáculos siendo los principales el acceso a la infraestructura tecnológica, así como la ausencia de competencias y pedagogías para la educación a distancia. Al mismo tiempo, se sugiere que el paso forzado a la enseñanza y el aprendizaje a distancia ofrece importantes oportunidades para proponer modelos de aprendizaje más flexibles, explorar el aprendizaje mixto o híbrido y para mezclar el aprendizaje sincrónico con el aprendizaje asincrónico. De modo similar también el Banco Mundial (World Bank, 2020) realiza una serie de recomendaciones de cómo enfrentarse a corto, medio y largo plazo desde las instituciones de educación terciaria a la nueva situación.

Con relación a la situación y panorama en Latinoamérica es relevante consultar el informe de UNESCO-ISEALC (2020) donde después de analizar el impacto en distintas regiones del continente, este organismo recomienda a las instituciones de educación superior acciones de política universitaria como:

- Anticiparse a una suspensión de larga duración, centrando los esfuerzos en asegurar la continuidad formativa y garantizar la equidad, generando mecanismos de gobierno, monitoreo y apoyo eficientes;

- Diseñar medidas pedagógicas para evaluar formativamente y generar mecanismos de apoyo al aprendizaje de los estudiantes en desventaja;

- Documentar los cambios pedagógicos introducidos y sus impactos;

- Aprender de los errores y escalar la digitalización, la hibridación y el aprendizaje ubicuo;

- Promover la reflexión interna sobre la renovación del modelo de enseñanza y aprendizaje

Por otra parte, existen publicaciones donde podemos consultar las propuestas o prospectivas ofrecidas por distintos expertos con relación al futuro de la educación superior en tiempos poscovid 19 (Peters y otros, 2020; Adedoyin, and Soykan, 2020; Rapanta, C., Botturi, L., Goodyear, P. et al. 2020). En su mayor parte estos trabajos coinciden en destacar que uno de los principales efectos de la Covid-19 sobre la

\footnotetext{
${ }^{1}$ En el contexto español, al inicio de la pandemia, el Ministerio de Universidades y CRUE (2020) crearon un portalweb de recursos para el aprendizaje no presencial denominado Conectad@s: la universidad en casa que fue diseñado y mantenido por la UNED y la UOC.
}

Análisis de las políticas de enseñanza universitaria en España para tiempos Covid19: la presencialidad adaptada. M. Area. A. Bethencourt, S. Martín y B. San Nicolás. 
RED. Revista de Educación a Distancia. Núm. 65, Vol. 21. Artíc. 3, 08-01-2021.

DOI: https://doi.org/10.6018/red.450461

enseñanza universitaria es que ésta se desplazará desde modelos totalmente presenciales hacia otros en línea y mixtos o semipresenciales.

El debate, a nivel mundial, sobre cómo las instituciones universitarias deben actuar y hacer frente ante los efectos de la pandemia está ofreciendo respuestas de política educativa bien diferenciadas entre las mismas. Así por ejemplo, algunas universidades han optado directamente por trasladar para el nuevo curso toda sus oferta de titulaciones y formativa a la modalidad de educación a distancia online, como es el caso de Cambridge o Manchester en Reino Unido o Harvard en EE.UU., mientras otras se afianzan en la defensa de la presencialidad como seña de su identidad docente por lo que articulan soluciones híbridas y muchas veces extrañas de combinación de lo físico con lo virtual, o simplemente no hacen nada y lo presencial es su única oferta.

\section{Problema y cuestiones del estudio}

En este artículo pretendemos explorar y analizar el concepto de "presencialidad adaptada" propuesto por el Ministerio de Universidades del Gobierno de España y asumido por el conjunto de equipos rectorales de las universidades presenciales españolas para la organización y desarrollo de la docencia en un contexto donde las medidas de seguridad sanitaria dificulten o impidan la asistencia presencial de todo el alumnado a las aulas universitarias. Un estudio con algunas similitudes, pero con diferentes objetivos, al aquí planteado fue desarrollado por Murphy (2020) donde analizó veinticinco declaraciones de elearning de emergencia en universidades estadounidenses.

En este sentido, lo primero que hemos de indicar, es que el concepto de presencialidad adaptada no existe en la bibliografía o literatura académica. Hemos realizado búsquedas en bases de datos y repositorios como Dialnet, Google Académico, Research Gate, ERIC, EBSCO y otras, y no hemos localizado ningún artículo o documento que incorpore este concepto ${ }^{2}$. Por lo que podemos afirmar que el mismo es una singularidad o particularidad española propuesto por una autoridad administrativa-política, pero no por la comunidad científica del campo de la Tecnología y la Educación.

Ante la ausencia de fundamentación teórica de esta propuesta, nos hemos propuesto diseccionar las recomendaciones y resoluciones que éstos han elaborado para la impartición de la docencia en el nuevo curso con la finalidad de indagar e identificar las medidas de actuación, así como las concepciones o supuestos pedagógicos que subyacen en dicha documentación con relación a la "presencialidad adaptada".

La preguntas o cuestiones que motivan este estudio y a las que queremos dar respuesta son:

- ¿Cuáles son las medidas o acciones de política docente propuestas por las universidades para implementar la presencialidad adaptada?

- ¿Existe consenso o disonancia entre las distintas universidades españolas con relación a sus políticas de presencialidad adaptada?

\footnotetext{
2 Únicamente localizamos algunos textos o pequeños ensayos reflexivos en el blog o revista digital The Conversation https://theconversation.com/es donde algunos expertos valoran las propuestas de organización docente dada en las universidades durante el Covid-19. En una de esas entradas o artículos Sánchez-Carracedo y otros (2020) nombran el concepto de "presencialidad adaptada" propuesto por el Ministerio de Universidades.
}

Análisis de las políticas de enseñanza universitaria en España para tiempos Covid19: la presencialidad adaptada. M. Area. A. Bethencourt, S. Martín y B. San Nicolás. 
RED. Revista de Educación a Distancia. Núm. 65, Vol. 21. Artíc. 3, 08-01-2021.

DOI: https://doi.org/10.6018/red.450461

- ¿Qué visiones pedagógicas y estratégicas subyacen a las políticas de las universidades españolas sobre la "presencialidad adaptada"?

\section{Metodología}

La metodología del estudio realizado se basa en la técnica de análisis de contenido documental. Las fuentes objeto de análisis fueron, por una parte, el documento con doce recomendaciones y un anexo con medidas de prevención e higiene frente a la Covid19 elaborado por el Ministerio de Universidades $(2020)^{3}$ y por otra, las resoluciones aprobadas por los equipos de gobierno de las diferentes universidades para el desarrollo de planes de contingencia ante la situación de alarma sanitaria por la Covid-194. Toda esta documentación fue publicada entre junio-julio 2020. Hemos de indicar que aunque toda la documentación analizada corresponde a las resoluciones elaboradas institucionalmente por las universidades públicas españolas, los documentos hacen referencia a temporalidades distintas ya que unos se elaboraron para el confinamiento, otros para afrontar el primer cuatrimestre de este curso y otros para todo el curso.

De las 50 universidades públicas españolas, la muestra para este estudio consistió en la selección de 20 de las mismas. El criterio básico fue seleccionar de modo aleatorio una universidad pública en cada comunidad autónoma, excepto en los casos de Andalucía, Cataluña y Madrid que se eligieron dos universidades ya que éstas son las comunidades con el mayor número de universidades públicas en su territorio.

De este modo se analizaron los documentos o resoluciones de las siguientes universidades: Universidad Autónoma de Barcelona, Universidad Autónoma de Madrid, Universidad Cantabria, Universidad Complutense de Madrid, Universidad de Castilla-La Mancha, Universidad de Extremadura, Universidad de Granada, Universidad de La Laguna, Universidad de La Rioja, Universidad de las Islas Baleares, Universidad de Murcia, Universidad de Oviedo, Universidad de Salamanca, Universidad de Santiago de Compostela, Universidad de Sevilla, Universidad de Valencia, Universidad de Zaragoza, Universidad del País Vasco, Universidad Pública de Navarra y Universidad Rovira i Virgili.

Para proceder al análisis de contenido de dichos documentos previamente se identificaron diez categorías de análisis o dimensiones que hemos aplicado sobre los mismos. Pueden verse en la tabla 1.

\footnotetext{
${ }^{3}$ Este documento puede consultarse en https://www.ciencia.gob.es/stfls/MICINN/Universidades/Ficheros/Recomendaciones_del_Minist erio_de_Universidades_para_adaptar_curso.pdf

${ }^{4}$ Estas resoluciones de las universidades agrupadas por comunidades autónomas puede consultarse en la Web del Ministerio de Universidades en https://bit.ly/2ldH3cS
}

Análisis de las políticas de enseñanza universitaria en España para tiempos Covid19: la presencialidad adaptada. M. Area. A. Bethencourt, S. Martín y B. San Nicolás. 
RED. Revista de Educación a Distancia. Núm. 65, Vol. 21. Artíc. 3, 08-01-2021.

DOI: https://doi.org/10.6018/red.450461

Tabla 1 Dimensiones o categorías de análisis del contenido

\begin{tabular}{|c|c|}
\hline Dimensiones & Descripción \\
\hline $\begin{array}{l}\text { Plan de contingencia } \\
\text { para la docencia online }\end{array}$ & $\begin{array}{l}\text { Dimensión que se refiere al Plan de contingencia de la } \\
\text { universidad en el que se establecen las pautas de } \\
\text { funcionamiento que deberán tenerse en cuenta durante el } \\
\text { curso, incluso si fuera necesario adoptar una modalidad } \\
\text { totalmente online. }\end{array}$ \\
\hline $\begin{array}{l}\text { Infraestructuras e } \\
\text { inversiones }\end{array}$ & $\begin{array}{l}\text { Esta dimensión se refiere a si se elaboran propuestas para la } \\
\text { mejora del equipamiento informático y redes telemáticas cara a } \\
\text { afrontar la presencialidad adaptada o una modalidad de } \\
\text { enseñanza totalmente en línea, así como posibles inversiones } \\
\text { previstas. }\end{array}$ \\
\hline $\begin{array}{l}\text { Seguridad personal de } \\
\text { la salud }\end{array}$ & $\begin{array}{l}\text { Dimensión referida a las medidas de seguridad sanitaria tanto } \\
\text { individuales como colectivas que deben adoptarse para } \\
\text { acceder a los centros y durante su estancia en los mismos. }\end{array}$ \\
\hline $\begin{array}{l}\text { Propuesta de } \\
\text { modalidad preferentes } \\
\text { de enseñanza } \\
\text { (presencial vs. virtual) }\end{array}$ & $\begin{array}{l}\text { Dimensión referida al tipo de modalidad de enseñanza que } \\
\text { asume y considera prioritaria la universidad, así como las } \\
\text { alternativas a la misma en caso de no fuera posible } \\
\text { implementarla. Incluye también las distintas formas o variantes } \\
\text { de interpretar la presencialidad adaptada en cada universidad. }\end{array}$ \\
\hline $\begin{array}{l}\text { Gestión de la } \\
\text { protección y derechos } \\
\text { de privacidad }\end{array}$ & $\begin{array}{l}\text { Dimensión sobre los aspectos relativos a las cuestiones } \\
\text { relacionadas con la protección de derechos, la privacidad o la } \\
\text { imagen tanto el alumnado como del profesorado en la red. }\end{array}$ \\
\hline $\begin{array}{l}\text { Competencia digital del } \\
\text { profesorado y } \\
\text { alumnado }\end{array}$ & $\begin{array}{l}\text { Dimensión sobre propuestas con relación a atender las } \\
\text { necesidades formativas para la mejora de la competencia } \\
\text { digital del profesorado y del alumnado como condición } \\
\text { necesaria para el desarrollo de las actividades académicas a } \\
\text { través de entornos digitales. }\end{array}$ \\
\hline
\end{tabular}

Análisis de las políticas de enseñanza universitaria en España para tiempos Covid19: la presencialidad adaptada. M. Area. A. Bethencourt, S. Martín y B. San Nicolás.

Página 6 de 19 
RED. Revista de Educación a Distancia. Núm. 65, Vol. 21. Artíc. 3, 08-01-2021.

DOI: https://doi.org/10.6018/red.450461

\begin{tabular}{|l|l|}
\hline $\begin{array}{l}\text { Disponibilidad de } \\
\text { espacios virtuales para } \\
\text { la docencia y sistemas } \\
\text { de apoyo a la docencia } \\
\text { virtual }\end{array}$ & $\begin{array}{l}\text { Dimensión referida al análisis y propuestas sobre la } \\
\text { disponibilidad de espacios virtuales que permitan la articulación } \\
\text { las salas de videoconferencia o las aulas virtuales. También } \\
\text { incluye si se ofertan servicios de apoyo institucionales para el } \\
\text { apoyo para este tipo de enseñanzas. }\end{array}$ \\
\hline $\begin{array}{l}\text { Visión de la política } \\
\text { educativa para la } \\
\text { transformación digital } \\
\text { posCovid-19 }\end{array}$ & $\begin{array}{l}\text { Dimensión referida al análisis de si se incorporan políticas, } \\
\text { reflexiones o propuestas de la universidad cara a } \\
\text { transformación e innovación digital de su sistema formativo a } \\
\text { medio o largo plazo ante la sociedad digital más allá de la } \\
\text { situación de emergencia sanitaria actual. }\end{array}$ \\
\hline $\begin{array}{l}\text { Atención a la brecha } \\
\text { digital }\end{array}$ & $\begin{array}{l}\text { Dimensión sobre el análisis que realiza la universidad con } \\
\text { relación a la brecha digital de su alumnado y si adoptan } \\
\text { medidas de compensación de esta situación que permita a los } \\
\text { estudiantes el seguimiento de las actividades no presenciales. }\end{array}$ \\
\hline aprendizaje & $\begin{array}{l}\text { Dimensión sobre cómo articulan las universidades los procesos } \\
\text { evaluativos en una modalidad de presencialidad adaptada o no } \\
\text { presencialidad. Se refiere a qué modalidad de evaluación } \\
\text { predomina y qué medidas adoptan las universidades para } \\
\text { garantizar estos procesos evaluativos. }\end{array}$ \\
\hline
\end{tabular}

En una primera fase se procedió a clasificar el contenido de las recomendaciones del Ministerio, así como de cada resolución o documento de las universidades seleccionadas en fichas individuales de cada caso. Posteriormente, se procedió a elaborar una matriz o cuadro comparativo donde figuraban horizontalmente dichas dimensiones y verticalmente el contenido que prescribía cada universidad en las mismas.

\section{Resultados}

A continuación, presentamos una síntesis de los principales resultados obtenidos en el análisis del contenido de los documentos o resoluciones según dichas dimensiones o categorías de análisis. 
RED. Revista de Educación a Distancia. Núm. 65, Vol. 21. Artíc. 3, 08-01-2021.

DOI: https://doi.org/10.6018/red.450461

\section{Plan de contingencia para la docencia online}

Una de las recomendaciones centrales del Ministerio de Universidades es que cada universidad elabore un plan de contingencia que permita un cambio masivo e inmediato a un sistema de docencia online en caso de una nueva oleada de la pandemia. Estos planes de contingencia deben especificarse en las adendas de cada plan de estudio y con la antelación a la apertura del periodo de matrícula para que la comunidad educativa pueda asumirlas sin afectar al bienestar de sus integrantes, así como a la calidad de la enseñanza ofertada. Por ese propósito, se especifica que la ANECA deberá acreditar la calidad de las modalidades de enseñanza adoptadas por las universidades.

En estos planes de contingencia, siguiendo las recomendaciones del Ministerio, debieran tener en cuenta la formación online del profesorado, la adaptación de sistemas de evaluación, el desarrollo de unas estrategias de digitalización para situaciones de emergencias que requiera la flexibilización de las modalidades de enseñanza, así como una concreción sobre los horarios de tutorías y seminarios, entre otras cuestiones. Así mismo, se recomienda que en cada centro universitario haya al menos una persona responsable relacionada con las cuestiones de gestión y actuación frente al COVID-19.

En las recomendaciones del Ministerio también se especifica que se fomentará la continuidad del teletrabajo para aquellas actividades que no requieren específicamente la presencialidad (tales como tutorías, reuniones, entre otras), así como el trabajo y las reuniones del propio profesorado en el que será de carácter no presencial siempre que sea posible. Otra de las cuestiones recurrentes dentro de las medidas necesarias por parte del Ministerio para las universidades, es la formación y educación en estas medidas de prevención, higiene y favorecimiento de la salud.

Pese a que la recomendación del Ministerio es que cada título universitario debe tener su propio plan de contingencia, se aprecia que los documentos elaborados por parte de las universidades tienen planteamientos y desarrollos distintos. En los documentos presentados, algunas universidades concretan las instrucciones en materia sanitaria para hacer frente a la pandemia, otras universidades dedican sus especificaciones en recomendación y orientación para el ejercicio de la docencia y el desarrollo del proceso de enseñanza y aprendizaje, y, otras, en cambio, han establecido directamente, un plan de contingencia con carácter general para toda la institución educativa.

Del análisis realizado podemos indicar que la mitad de las instituciones analizadas contemplan en su documentación la creación de diferentes escenarios (con medidas específicas y modalidades de enseñanza distintas) según la situación sanitaria y las exigencias por parte de las autoridades sanitarias. Básicamente se contemplan tres potenciales escenarios: la modalidad de absoluta presencialidad, la modalidad semipresencial en caso de que se tenga que reducir el aforo por las distancias de seguridad y la modalidad no presencial o a distancia en caso de que se produzca una nueva situación de confinamiento o se tenga que aislar grupos o clases y no sea posible la presencialidad. Estos son los casos de la Universidad de La Laguna, de Murcia, La Rioja, Complutense de Madrid, Autónoma de Madrid, Sevilla, Salamanca, Extremadura, Santiago de Compostela y La Rioja.

Asimismo, pese a que el Ministerio pida la elaboración de planes de contingencia por cada centro y para cada titulación, no todas las universidades contemplan, según su documentación, esta especificidad en sus medidas organizativas. Menos de la mitad analizada recoge los pasos necesarios para la creación de estos documentos por centro.

Análisis de las políticas de enseñanza universitaria en España para tiempos Covid19: la presencialidad adaptada. M. Area. A. Bethencourt, S. Martín y B. San Nicolás. 
RED. Revista de Educación a Distancia. Núm. 65, Vol. 21. Artíc. 3, 08-01-2021.

DOI: https://doi.org/10.6018/red.450461

\section{Infraestructuras e inversiones}

En muchas instituciones, no se contemplan o no se especifican los gastos e inversiones que deben realizar las universidades o recogen lo indicado en la recomendación del Ministerio de Universidades que concreta que se realizará la correspondiente transferencia presupuestaria a las Comunidades Autónomas para afrontar las necesidades derivadas de la situación de emergencia sanitaria.

Las Universidades que si hablan de las necesidades de inversión para afrontar las necesidades derivadas de la situación sanitaria durante el curso 2020-2021, lo hacen para referirse principalmente a la dotación, a la formación y la compensación de la brecha digital. Solo en el caso de la Universidad de Murcia se habla de los costes derivados de los recursos humanos, indicando que no disponen de financiación para asumir nuevas necesidades docentes.

\section{Seguridad personal de la salud}

Este es uno de los contenidos centrales de las recomendaciones realizadas por parte del Ministerio de Universidades desde el cual se señala la necesidad de garantizar la salud personal del profesorado, alumnado y personal de servicios durante la pandemia del Covid19. En dichas recomendaciones establecen medidas concretas de naturaleza sanitaria como las siguientes:

- Garantía en el número de estudiantes en la instalación no supere la capacidad de la misma teniendo en cuenta la separación de 1,5 metros entre los ocupantes, y en caso de no poderse garantizar, no desarrollar una docencia presencial.

- Reducción de grupos y desdobles para garantizar el distanciamiento social

- Uso de sistemas de streaming para transmisión de las sesiones de clase cuando no se posibilite lo anterior.

- Higiene de manos de forma frecuente y uso geles hidroalcohólicos.

- Uso obligatorio de la mascarilla para todas las personas que accedan al centro y en todos los espacios del centro, durante la permanencia en el mismo, siguiendo las recomendaciones de la autoridad sanitaria y la normativa vigente en cada Comunidad Autónoma, e insistiendo en su correcta utilización.

- Refuerzo de las tareas de limpieza y desinfección de espacios.

- Prohibición del acceso a los centros a aquellas personas con síntomas compatibles con Covid-19.

Todas las universidades españolas toman estas pautas como referencia y en su mayoría las siguen sin alteración alguna incluyéndose en sus resoluciones para el nuevo curso. En algún caso, algunas universidades desarrollan con mayor detalle y añaden especificidades sanitarias en sus planes de contingencia.

\section{Propuesta de modalidad preferente de enseñanza (presencial vs. virtual)}

El documento marco del Ministerio hace énfasis en la necesidad de utilizar la experiencia obtenida a finales del curso 2019 - 2020 durante los meses de confinamiento en los hogares, el cual supuso un traslado de toda la docencia a modalidad online y cerrar el curso alejados de las aulas y las facultades. Recoge que es de interés para esta nueva etapa las vivencias y experiencias tanto referidas a la enseñanza como a la evaluación no presencial por parte de los docentes y alumnado, para evaluar y desarrollar la capacidad de las universidades españolas para operar en un entorno digital, aplicando nuevas formas interacción en aquellas actividades en las que sea viable y se puedan beneficiar de dichas metodologías.

Sin embargo, a pesar de apoyar a la comunidad universitaria para un desempeño óptimo en el terreno digital y abordar con éxito el reto de la no-presencialidad, el Ministerio de

Análisis de las políticas de enseñanza universitaria en España para tiempos Covid19: la presencialidad adaptada. M. Area. A. Bethencourt, S. Martín y B. San Nicolás. 
RED. Revista de Educación a Distancia. Núm. 65, Vol. 21. Artíc. 3, 08-01-2021.

DOI: https://doi.org/10.6018/red.450461

Universidades remarca la necesidad de mantener la presencialidad de la universidad: "reiteramos la deseable preponderancia de la enseñanza presencial como forma más adecuada para la educación superior de calidad en términos generales." Es de destacar, en este sentido, cómo en el discurso se asocia la calidad de la enseñanza a la modalidad presencial. Reafirmando lo anterior, en el mismo documento se establece la deseabilidad de poder compatibilizar actividades de enseñanza presenciales con otras de carácter online, permitiendo así el desarrollo de un modelo mixto "presencial-no presencial".

A grandes rasgos, los documentos y resoluciones publicados por cada universidad para adaptar la docencia en el presente curso escolar siguen fielmente las indicaciones del Ministerio sobre la presencialidad adaptada, sin embargo, las interpretaciones de algunas universidades son más flexibles y permiten la incorporación de actividades asíncronas o de modalidades semipresenciales con variantes y aplicables según sea necesario.

La mayoría de las universidades recalca que el carácter de éstas es presencial y que las medidas a tomar en modalidad semipresencial u online se plantean únicamente en el marco de la pandemia por Covid-19 que requiere del desarrollo de planes de contingencia y medidas especiales para poder adaptar las tareas de enseñanza y aprendizaje para su desarrollo óptimo más allá de los espacios físicos de la facultades y centros. Las comisiones de cada universidad atienden a las medidas sanitarias generales como indicador de cómo y cuándo proceder con estos planes.

Muchos centros coinciden en la necesidad de diseñar un plan que contemple varios escenarios de actuación. Así, es común encontrar diferentes contextos que llevan implícitos un cambio en la modalidad o en las medidas preventivas. Las universidades de Sevilla, Islas Baleares, La Laguna, Autónoma de Madrid y Santiago de Compostela, apuestan por esta estrategia. Todas ellas establecen que su modalidad preferente es la presencial y en muchos casos se pretende iniciar así el curso académico 2020-21, aunque contemplan volver a una docencia totalmente online o parcialmente online según las exigencias sanitarias.

La Universidad de Sevilla indica que se contempla la presencialidad adaptada inicialmente y prevé dos posibles escenarios: un escenario de menor actividad académica presencial como consecuencia de medidas sanitarias de distanciamiento interpersonal que limiten el aforo permitido en las aulas (escenario A) y un escenario de suspensión de la actividad presencial (escenario B). El caso de la Universidad de Islas Baleares expone dos posibles escenarios en función de la evaluación de la pandemia: escenario 1, donde se daría un cierre total de la UIB con todas las actividades presenciales se transformarán en actividades en línea no presenciales; y un escenario 2 , con un cierre parcial por edificios. En este caso prevalece también la presencialidad. Otras universidades como la de Santiago de Compostela y la de La Laguna, indican el deseo de respetar el carácter presencial de la docencia, y organizan sus escenarios en función de esta premisa, llegando a contemplar tres posibles momentos o escenarios.

La Universidad de Salamanca indica entre sus medidas organizativas que siempre que las condiciones sanitarias lo permitan, la docencia del curso 2020-2021 se va a caracterizar por una «presencialidad segura». Las universidades de Castilla-La Mancha, La Rioja, Granada, Cantabria, Zaragoza, Extremadura, País Vasco, Rovira i Virgili y la Universidad Pública de Navarra también recogen medidas de seguridad para la vuelta a la actividad presencial.

La Universidad de Oviedo también estipula una docencia presencial a priori, aunque estableciendo las características que marcarían una docencia semipresencial y online.

Análisis de las políticas de enseñanza universitaria en España para tiempos Covid19: la presencialidad adaptada. M. Area. A. Bethencourt, S. Martín y B. San Nicolás. 
RED. Revista de Educación a Distancia. Núm. 65, Vol. 21. Artíc. 3, 08-01-2021.

DOI: https://doi.org/10.6018/red.450461

Por ejemplo, se adoptará el nivel de presencialidad que la situación requiera. Como regla, se establece que la presencialidad mínima sea del $80 \%$. En Murcia, debido a las instalaciones físicas y los tamaños de los grupos y la capacidad de las clases, en las titulaciones de Grados la docencia no se llevará a cabo de forma presencial; como sí ocurre en las titulaciones de Máster, donde los grupos son más reducidos.

La Universidad Autónoma de Barcelona, identifica la modalidad de la universidad como presencial y se plantea durante el presente curso una modalidad mixta dada la situación de emergencia sanitaria combinado actividades formativas presenciales y no presenciales. Dada la situación de incertidumbre se mantienen abiertas las posibilidades de que durante el curso cambie la situación actual, mejorando o empeorando, por lo que podría plantearse una modalidad $100 \%$ presencial y una modalidad $100 \%$ no presencial. Se prioriza la actividad presencial, especialmente la del alumnado de primer curso y de último curso. En el caso de los másteres se podrá plantear su desarrollo en una modalidad $100 \%$ no presencial.

\section{Gestión de la protección y derechos de privacidad digital}

En las recomendaciones generales del Ministerio de Universidades no se aprecia ninguna mención directa o indirecta sobre la gestión de la protección y los derechos de privacidad digital. Precisamente por ello, es posible que, por ese mismo motivo, el listado de universidades que no contemplen esta categoría sea bastante amplio (Universidades como la de Granada, Zaragoza, Islas Baleares, Autónoma de Barcelona, Castilla-La Mancha, Rovira i Virgili, Autónoma de Madrid, Valencia, Cantabria, Extremadura, Santiago de Compostela, La Rioja, Murcia y la Universidad Pública de Navarra).

Sin embargo, algunas universidades (Complutense de Madrid, Salamanca, País Vasco, Sevilla, Oviedo, La Laguna entre otras) han explicitado algunas medidas pertinentes a la protección de imagen, a los derechos de protección intelectual y de privacidad de los usuarios.

\section{Competencia digital del profesorado y alumnado}

El Ministerio de Universidades contempla como necesario que el profesorado de los centros cuente con las habilidades necesarias para la aplicación óptima de los planes de contingencia diseñados. En este sentido, se establece como elemento obligatorio que debe estar presente en estas medidas propias de cada universidad, el establecimiento de planes de formación del profesorado en la enseñanza online, tanto en competencias digitales como en nuevas metodológicas que posibiliten una educación semipresencial u online. En el caso de formaciones específicas para el desarrollo de la competencia digital del alumnado universitario no se hace tan notable.

La mayoría de las universidades analizadas en este estudio cumple con lo solicitado y en sus planes se encuentra información relacionada a la formación docente. Así, las universidades de Sevilla, Granada, Zaragoza, Oviedo, La Laguna, Salamanca, Complutense de Madrid, Autónoma de Madrid, Valencia, Extremadura, Santiago de Compostela, y la Autónoma de Barcelona contemplan una ampliación de su Plan de Formación del Profesorado en la que se dé respuesta a las necesidades con diversas acciones formativas centradas en la competencia digital docente y el manejo de herramientas digitales. Estas formaciones se ofertan en su mayoría en formato de curso online o webinars.

Las universidades de Islas Baleares, Cantabria, Castilla-La Mancha, La Rioja, Murcia, Universidad Pública de Navarra, País Vasco, y Rovira i Virgili no hacen referencia alguna al desarrollo de la competencia digital en sus planes.

Análisis de las políticas de enseñanza universitaria en España para tiempos Covid19: la presencialidad adaptada. M. Area. A. Bethencourt, S. Martín y B. San Nicolás. 
RED. Revista de Educación a Distancia. Núm. 65, Vol. 21. Artíc. 3, 08-01-2021.

DOI: https://doi.org/10.6018/red.450461

En cuanto a la formación específica para el alumnado universitario, sólo algunas contemplan el desarrollo de acciones formativas en el manejo de herramientas digitales para el aprendizaje. Este es el caso de la Universidad de Granada, la cual proporciona el espacio virtual colaborativo "COVID-19: Adaptación a la docencia y evaluación no presencial", asistido por voluntariado experto en informática de manera que se abran foros de discusión en los que el estudiantado resuelva dudas relacionadas con la adaptación a las nuevas estrategias de docencia y evaluación no presencial. En la Universidad de Salamanca y Valencia también atienden a la necesidad de ofertar cursos específicos para el alumnado en el manejo de las aplicaciones universitarias.

\section{Disponibilidad de espacios virtuales y sistemas de apoyo a la docencia en línea}

El marco que recoge las orientaciones generales para los planes de contingencia de las universidades españolas contempla como necesario la disponibilidad de espacios virtuales que apoyen la docencia tanto semipresencial, en línea o en "presencialidad adaptada". En este sentido las recomendaciones se concretan en la necesidad de contar con sistemas informáticos que permitan la retransmisión de las sesiones de clase vía streaming cuando las necesidades lo demanden.

En sus resoluciones, la gran parte de las universidades recoge el uso de estos sistemas de emisión además de entornos virtuales de aprendizaje que ya muchas venían usando en su normalidad preCovid-19. Algunas de ellas detallan las plataformas y herramientas que utilizarán en el curso 2020-2021.

\section{Política educativa para transformación digital de la enseñanza poscovid-19}

En las recomendaciones generales del Ministerio de Universidades se concreta que cada universidad debe tener un plan de contingencia que permita un cambio masivo e inmediato a un sistema de docencia online, sin embargo, se especifica que se hará solo en el caso que la situación sanitaria así lo requiera. Por lo tanto, no contempla una visión de sostenibilidad futura en relación con la apuesta hacia la docencia online ni a la docencia semipresencial. De este modo, la presencialidad adaptada, así como las modalidades semipresenciales $u$ online son consideradas necesarias siempre y cuando la situación sanitaria lo exija.

En el documento del Ministerio se señala que la experiencia desarrollada en docencia y evaluación no presencial (docencia online) durante los meses del confinamiento debiera ser aprovechada para favorecer la flexibilidad en la incorporación de la enseñanza digital, aunque se reitera en la deseabilidad predominante de la enseñanza presencial para la educación superior.

Con respecto a esta categoría o dimensión de análisis, son muy pocas las universidades que incluyen alguna mención sobre política educativa tras la pandemia. Esto pone en evidencia que las universidades españolas han elaborado dichas resoluciones presionadas por el carácter urgente e inmediato de respuesta ante la pandemia y no tanto con la intencionalidad de abrir el debate sobre la transformación digital de las universidades. En otras palabras, las resoluciones elaboradas por los equipos de gobierno de las universidades españolas están impulsadas por la emergencia sanitaria sin que exista, al menos de forma visible, una estrategia de digitalización a medio y largo plazo de educación superior.

\section{Evaluación del aprendizaje del alumnado}

En cuanto a la dimensión relativa a la de evaluación del aprendizaje, el Ministerio de Universidades especifica en su documento que la heterogeneidad de las posibles tareas educativas complejiza unas recomendaciones generales sobre la evaluación del

Análisis de las políticas de enseñanza universitaria en España para tiempos Covid19: la presencialidad adaptada. M. Area. A. Bethencourt, S. Martín y B. San Nicolás. 
RED. Revista de Educación a Distancia. Núm. 65, Vol. 21. Artíc. 3, 08-01-2021.

DOI: https://doi.org/10.6018/red.450461

aprendizaje en la docencia universitaria. Sin embargo, se recomienda que en los planes de contingencia aparezcan los sistemas de evaluación adaptados que se realizarán en las diferentes titulaciones. El Ministerio declara que el conocimiento experiencial adquirido en la fase del confinamiento puede favorecer la utilización de estrategias en modalidad online para fines didácticos y evaluativos, aunque se reitera la necesidad de la presencialidad tanto para la docencia como para la evaluación del aprendizaje.

Siguiendo las recomendaciones del Ministerio, muchas instituciones universitarias coinciden en priorizar o recomendar la presencialidad en la evaluación. Por ese mismo motivo, en las Universidades que recogen un apartado específico para la evaluación o que, se menciona a lo largo del discurso especifican que, siempre que las condiciones sanitarias así lo permitan, las pruebas de evaluación de las asignaturas se realizarán preferentemente de forma presencial.

Las universidades que priorizan la presencialidad en la evaluación también asumen su posible adaptación a los posibles cambios de escenarios. En los escenarios donde la presencialidad esté asegurada ya sea con las restricciones sanitarias o por el fin de la pandemia, la evaluación en estos escenarios será presencial. En los escenarios donde se desarrolle la semipresencialidad algunas instituciones universitarias recomiendan, del mismo modo, la presencialidad para las pruebas finales de las asignaturas que así lo contemplen. Por el contrario, otras universidades recomiendan preferentemente la realización de estas pruebas con carácter telemático o que, por otro lado, se puede optar a sustituir por otras actividades de evaluación que el alumnado pueda llevar a cabo y no requiera de presencialidad. En el caso de la Universidad de las Islas Baleares, por ejemplo, el profesorado podrá optar por hacer exámenes en línea, pese a que los exámenes se programarán de manera presencial. De modo similar, universidades como Santiago de Compostela, Extremadura, La Laguna o la Complutense de Madrid, señalan que si se produce una nueva situación de confinamiento se podrán realizar pruebas de carácter telemático u online.

Independientemente de la realización de estas pruebas finales en el plazo de la convocatoria, muchas instituciones universitarias especifican que las modalidades de evaluación deben ser íntegramente, preferentemente 0 de forma combinada metodologías evaluativas continuas y formativas (Autónoma de Madrid, Valencia, Complutense de Madrid, Murcia, Granada, La Laguna, Salamanca, Santiago de Compostela, País Vasco, Zaragoza). En algunos casos, se avanza un poco más en la "recomendación" y se sugiere que se valore el prescindir el examen (caso de Universidad Granada) o que se proponga una alternativa en la evaluación (Universidad del País Vasco).

\section{Atención a brecha digital}

En cuanto a la atención a la brecha digital, el Ministerio de Universidades recomienda que las universidades públicas deben asegurar la conectividad del conjunto del estudiantado para evitar la brecha digital y su posible continuidad. En otro apartado dentro de las mismas recomendaciones se reitera que en el desarrollo del curso 2020/2021, se debe garantizar todos los medios necesarios a la comunidad educativa de forma equitativa.

De este modo, las universidades han planteado tomar medidas dirigidas a paliar la brecha digital existente entre el alumnado para favorecer la conectividad o el acceso a los recursos tecnológicos. En concreto, entre los documentos analizados, se especifica que las Universidades Autónomas de Madrid y Barcelona, así como las Universidades del País Vasco, Oviedo y Sevilla favorecerán la adaptación de la enseñanza y la

Análisis de las políticas de enseñanza universitaria en España para tiempos Covid19: la presencialidad adaptada. M. Area. A. Bethencourt, S. Martín y B. San Nicolás. 
RED. Revista de Educación a Distancia. Núm. 65, Vol. 21. Artíc. 3, 08-01-2021.

DOI: https://doi.org/10.6018/red.450461

evaluación del alumnado con dificultades económicas o necesidades específicas de apoyo educativo para favorecer la igualdad de oportunidades.

Por otro lado, con respecto a las medidas específicas de dotación económica, las universidades de Valencia, Granada, y Sevilla impulsarán los planes de becas y ayudas propias que tenga en cuenta el efecto de la crisis y la dotación de equipos informáticos y tarjetas de conexión a internet. La Universidad de La Laguna también informará al alumnado la existencia de estas ayudas en la institución para el alumnado que lo requiera, además añade que en las pruebas de evaluación en línea se respetará la igualdad de acceso. Las universidades de Castilla-La Mancha, Complutense de Madrid y la del País Vasco apuestan por las medidas a favor de la dotación de dispositivos tecnológicos o a la reorganización de su servicio de préstamos de ordenadores y apoyo a la conexión a Internet.

Las Universidades de Rovira i Virgili, Extremadura, Murcia, La Rioja, Salamanca, Cantabria y las Islas Baleares, no especifican ningún apartado con relación a la brecha digital.

\section{Conclusiones}

Una primera evidencia es que el documento de recomendaciones propuesto por el Ministerio de Universidades ha sido utilizado por la mayor parte de los equipos de gobierno universitarios como marco de referencia para la elaboración de las resoluciones particulares para su institución. Esto nos permite concluir que, al menos en esta ocasión, existe un cierto nivel de coordinación. similitud o coincidencia de política universitaria similar en el conjunto del sistema universitario español ante el incierto curso 2020-21 condicionado por la pandemia del Covid19.

Una segunda conclusión es que, tanto desde el Ministerio de Universidades como de los equipos de gobierno de las universidades públicas mantienen una visión estratégica de defensa de la enseñanza presencial. Solo prevén o justifican la digitalización de sus formas organizativas por motivos sanitarios, estando totalmente ausentes los pedagógicos. En este sentido concluimos que, en los documentos tanto del Ministerio como de los rectorados, subyace una concepción de la pedagogía universitaria anclada en los tiempos predigitales, aferrada a un modelo organizativo de la enseñanza basada en el encuentro físico, concurrente o presencial entre el profesorado y estudiantes. Es más, en aquellos casos, donde se sugiere la digitalización de la enseñanza se impulsan medidas apoyadas en la telepresencia 0 encuentro sincrónico mediado telemáticamente. Ejemplos de ello son la instalación de cámaras en las clases presenciales para retransmitir en remoto dicha situación para que sea vista en tiempo real por alumnado que no pueda acudir físicamente al aula. O la realización de videoconferencias entre docente y alumnado en los horarios rígidos y establecidos oficialmente para cada asignatura tal como si fuera el horario de clases presenciales.

Las universidades públicas españolas siguen apoyándose más en la tradición didáctica de la enseñanza presencial que en los retos de innovación educativa que supone la enseñanza digital. Ninguna se atreve a romper o cuestionar los modelos rígidos de la organización del tiempo académico y de los métodos seculares de la enseñanza universitaria. En el actual contexto de la pandemia las instituciones de educación superior deberían aprovechar esta oportunidad para abrir y/o impulsar modelos pedagógicos que enfaticen más la autonomía y autorregulación del alumnado como sujeto protagonista de su aprendizaje, en la flexibilización de los espacios y horarios a la hora de implementar la docencia en los centros y facultades, en la reformulación de

Análisis de las políticas de enseñanza universitaria en España para tiempos Covid19: la presencialidad adaptada. M. Area. A. Bethencourt, S. Martín y B. San Nicolás. 
RED. Revista de Educación a Distancia. Núm. 65, Vol. 21. Artíc. 3, 08-01-2021.

DOI: https://doi.org/10.6018/red.450461

la oferta sus titulaciones para hacerlas más atractivas y adaptables a las necesidades y características del alumnado de la sociedad digital, para potenciar nuevas funciones y tareas docentes acordes con los tiempos del teletrabajo, entre otras cuestiones.

En tercer lugar, el concepto de la presencialidad adaptada, como ya señalamos anteriormente, no existe en la bibliografía académica. Esto ocurre, como ya indicamos anteriormente, porque es un concepto propuesto desde instancias políticoadministrativas de modo urgente y coyuntural ante la pandemia. No es un concepto derivado o apoyado en el conocimiento e investigación internacional de este campo. En este sentido, aunque no se reconozca explícitamente en dichas resoluciones, el concepto de presencialidad adaptada representa una variante o versión española del concepto de enseñanza semipresencial o blended learning que tiene una tradición de casi dos décadas en el panorama internacional (Bielawski and Metcalf, 2003; Garrison and Vaughan, 2008; Keengwe and Agamba, 2015; Tucker, Wycoff and Green, 2016; Cheung et al., 2018).

La singularidad a la que aludimos se refiere a que el concepto de presencialidad adaptada desarrollado por las universidades españolas consiste, en la mayor parte de los casos, en trasladar miméticamente el horario de las clases presenciales a un modelo de retransmisión online de las mismas. El objetivo preferente de dichas resoluciones busca garantizar el sincronismo temporal entre el profesorado y el alumnado bien in situ en los espacios físicos, bien telemáticamente en tiempo real a través de videoreuniones. Se pretende que tanto el profesorado como el alumnado cumplan el horario de las clases presenciales, es decir que pasen juntos determinado número de horas bien sea de forma empírica o de forma virtual.

Bajo este planteamiento subyace una concepción pedagógica de la enseñanza universitaria basada en un modelo expositivo o transmisivo del conocimiento. La presencialidad adaptada, tal como se formula, parece ir dirigido a reforzar aquellas prácticas de docencia apoyadas en la lección magistral del profesor -sea en el aula física o bien telemáticamente- y por otra, desconsidera como tiempo y espacio educativo el trabajo autónomo de los estudiantes así como la interacción asíncrona que ocurre a través de las aulas o espacios virtuales de enseñanza-aprendizaje. Consideramos que este enfoque o propuesta representa una distorsión de las características y principios pedagógico-organizativos del blended learning o enseñanza semipresencial (Area, 2020). En otras palabras, las políticas universitarias planificadas ante la pandemia no tuvieron en cuenta las aportaciones del conocimiento académico formulado en estos últimos años por la comunidad internacional experta en el eLearning y la Tecnología Educativa (Sangrá, 2020).

En cuarto lugar, podemos sugerir que casi todas las resoluciones y/o recomendaciones realizadas tanto por el Ministerio de Universidades como los gobiernos de las universidades españolas han tenido una visión cortoplacista dirigida a dar respuesta inmediata a los problemas causados por la pandemia. Son resoluciones condicionadas por criterios sanitarios. En este contexto de emergencia era necesario e imprescindible, pero carecen de visión estratégica a medio y largo plazo. Esto se evidencia en que las universidades públicas españolas, al menos en función de la documentación analizada, no plantean propuestas sobre la transformación de la enseñanza universitaria en la sociedad digital. Carecen de una visión estratégica posCovid-19.

En otras palabras, ha sido una ocasión desaprovechada para plantear y construir una visión estratégica para la adaptación o reconversión de los actuales estudios universitarios españoles hacia modelos semipresenciales o híbridos de enseñanza. La transformación digital de la docencia universitaria es una necesidad histórica para dar

Análisis de las políticas de enseñanza universitaria en España para tiempos Covid19: la presencialidad adaptada. M. Area. A. Bethencourt, S. Martín y B. San Nicolás. 
RED. Revista de Educación a Distancia. Núm. 65, Vol. 21. Artíc. 3, 08-01-2021.

DOI: https://doi.org/10.6018/red.450461

respuesta adecuada a las demandas y necesidades de la sociedad digital. No es solo una necesidad coyuntural de los tiempos de pandemia.

No obstante, y pese a lo anterior, creemos que la experiencia desarrollada en la fase del confinamiento, así como las nuevas formas de presencialidad adaptada que se implementarán durante el curso 2020-21 puede acelerar, en un futuro próximo, algunos cambios relevantes en las políticas educativas en el sistema universitario español. El conjunto de estas resoluciones es un primer paso institucional.

Por otra parte, durante la pandemia el conjunto del alumnado y el profesorado ha vivenciado la experiencia de enseñar y aprender telemáticamente desde casa y los equipos de gobierno han tomado conciencia del fenómeno y potencialidades de la enseñanza digital. Este hecho seguramente podrá favorecer actitudes más predispuestas y abiertas a reformular las titulaciones, hasta ahora exclusivamente ofertadas presencialmente, hacia modalidades de enseñanza híbridas, mezcladas 0 semipresenciales. Probablemente también se impulsará institucionalmente la oferta de nuevas titulaciones a distancia ofertadas desde las universidades presenciales de nuestro país. Los resultados obtenidos nos indican que las condiciones infraestructurales y de servicios ya existentes junto con la mejora de las competencias digitales del profesorado y el alumnado harán posible todos estos cambios.

Otra conclusión tiene que ver con la limitada atención que las universidades públicas realizan hacia la brecha digital en los estudiantes ya que, salvo en pocos casos, no se especifica detalladamente las características de estas medidas. Las referencias son en muchos casos meramente nominales y epidérmicas. De forma similar creemos que es preocupante que la mayor parte de las universidades públicas españolas, al igual que ocurre con las recomendaciones del Ministerio, no contemplan ningún apartado ni mención sobre la gestión de la protección de los datos digitales y los derechos de privacidad de su profesorado y alumnado. Ambos fenómenos deben ser abordados por cualquier política universitaria destinada a favorecer la igualdad de oportunidades educativas y la protección de los derechos de ciudadanía digital.

Para finalizar hemos de señalar que la principal limitación de este estudio es que ha focalizado su análisis sobre el contenido o semántica de lo declarado en resoluciones y documentos oficiales. Hemos realizado, lo que se denomina, un análisis del discurso de la política educativa. Es una contribución relevante, pero insuficiente. Sugerimos continuar con investigaciones empíricas sobre la ejecución de dichas políticas en cada universidad desarrollando estudios multicaso donde se analicen las acciones implementadas o llevadas a la práctica (inversiones tecnológicas, nuevos reglamentos, formación profesorado, guías docentes, servicios de apoyo, reformulación de los planes de organización docente, rediseño de las titulaciones,...) en cada contexto institucional.

Presentación del artículo: 14 de octubre de 2020

Fecha de aprobación: 9 de noviembre de 2020

Fecha de publicación: 08 de enero de 2021

Area-Moreira, M., Bethencourt-Aguilar, A., Martín-Gómez, S. y San Nicolás-Santos, B. (2020). Análisis de las políticas de enseñanza universitaria en España en tiempos de Covid-19. La presencialidad adaptada. RED. Revista Educación a Distancia, 21(65). https://doi.org/10.6018/red.450461

Análisis de las políticas de enseñanza universitaria en España para tiempos Covid19: la presencialidad adaptada. M. Area. A. Bethencourt, S. Martín y B. San Nicolás.

Página 16 de 19 
RED. Revista de Educación a Distancia. Núm. 65, Vol. 21. Artíc. 3, 08-01-2021.

DOI: https://doi.org/10.6018/red.450461

\section{Financiación}

Este trabajo no ha recibido ninguna subvención específica de los organismos de financiación en los sectores públicos, comerciales o sin fines de lucro.

\section{Referencias}

Adedoyin, O.B. and Soykan, E. (2020). Covid-19 pandemic and online learning: the challenges and opportunities, Interactive Learning Environments, Interactive Learning Environments DOI: 10.1080/10494820.2020.1813180 https://www.tandfonline.com/doi/full/10.1080/10494820.2020.1813180

Area, M. (2020). La enseñanza semipresencial. Mezclando lo presencial y lo virtual. En M. Turull (Coord): Manual de enseñanza universitaria. Barcelona, OctaedroICE/UB, pg. 259-270. http://diposit.ub.edu/dspace/bitstream/2445/166737/1/15213Manual-de-docencia-universitaria-FINAL.pdf

Bielawski, L. and Metcalf, D. (2003). Blended E-Learning. Integrating Knowledge, Performance, Support and Online Learning. Massachusetts, HRD Press.

Crawford, J. y otros (2020). COVID-19: 20 countries' higher education intra-period digital pedagogy responses. Journal of Applied Learning \& Teaching JALT, 3 (1) https://doi.org/10.37074/jalt.2020.3.1.7

Cheung, S.K.S,; Kwok,L.; Kubota,K; Lee, L.and Tokito, J. (2018): Blended Learning. Enhancing Learning Success. 11th International Conference, ICBL 2018, Osaka, Japan, Springer International Publishing.

Garrison, D.R. and Vaughan, N.D. (2008). Blended Learning in Higher Education: Framework, Principles, and Guidelines. San Francisco, Jossey-Bass

Gouëdard, P., Pont, B. and R. Viennet (2020). Education responses to COVID-19: shaping an implementation strategy. OECD Education Working Papers, No. 224, https://doi.org/10.1787/8e95f977-en

Keengwe, J. and Agamba, J.J. (2015). Models for Improving and Optimizing Online and Blended Learning in Higher Education. Hershey. PA, USA. IGI Publishing.

Marinoni, G.; Land, H. and Jensen, T (2020). The Impact Of Covid-19 on Higher Education around the World. IAU Global Survey Report. International Association of Universities (IAU) https://www.iauaiu.net/IMG/pdf/iau_covid19_and_he_survey_report_final_may_2020.pdf

Ministerio de Universidades (2020). Recomendaciones del Ministerio de Universidades a la comunidad universitaria para adaptar el curso universitario 2020-2021 a una presencialidad adaptada y medidas de actuación de las universidades ante un caso sospechoso o uno positivo de Covid-19. Ministerio de Universidades del Gobierno de España. Disponible en https://www.ciencia.gob.es/stfls/MICINN/Universidades/Ficheros/Recomendaciones _del_Ministerio_de_Universidades_para_adaptar_curso.pdf

Análisis de las políticas de enseñanza universitaria en España para tiempos Covid19: la presencialidad adaptada. M. Area. A. Bethencourt, S. Martín y B. San Nicolás. 
RED. Revista de Educación a Distancia. Núm. 65, Vol. 21. Artíc. 3, 08-01-2021.

DOI: https://doi.org/10.6018/red.450461

Ministerio de Universidades/CRUE (2020).Conectad@s: La Universidad En Casa. Portalweb de recursos disponible en https://www.uned.es/universidad/inicio/uned_uoc_solidaria.html

Murphy, M. (2020). COVID-19 and emergency eLearning: Consequences of the securitization of higher education for post-pandemic pedagogy. Contemporary Security Policy, Advanced Online Publication.

https://www.tandfonline.com/doi/full/10.1080/13523260.2020.1761749

OECD and Harvard Graduate School of Education (2020). A framework to guide an education response to the COVID-19 Pandemic of 2020.

https://globaled.gse.harvard.edu/files/geii/files/framework_guide_v2.pdf

Peters M. A.y otros (2020). Reimagining the new pedagogical possibilities for universities post-Covid-19. Journal Educational Philosophy and Theory. https://www.tandfonline.com/doi/full/10.1080/00131857.2020.1777655

Rapanta, C., Botturi, L., Goodyear, P. et al. (2020). Online University Teaching During and After the Covid-19 Crisis: Refocusing Teacher Presence and Learning Activity. Postdigital Science Education https://link.springer.com/article/10.1007/s42438-02000155-y

Reimers, F. y Schleicher, A. (2020흘. Schooling disrupted, schooling rethought. How the Covid-19 pandemic is changing education. OCDE, Global Education Innovation Initiative.

https://globaled.gse.harvard.edu/files/geii/files/education_continuity_v3.pdf

Reimers, F. y Schleicher, A. (2020b). A framework to guide an education response to the COVID-19 Pandemic of 2020. OECD. https://read.oecd-

ilibrary.org/view/?ref=126_126988-t63lxosohs\&title=A-framework-to-guide-aneducation-response-to-the-Covid-19-Pandemic-of-2020

Sánchez-Carracedo, F. et al. (2020). La universidad que viene: de la 'docencia remota de emergencia' a la 'presencialidad adaptada'. The Conversation http://hdl.handle.net/2117/328629

Sangrá, A. (Coord.) (2020). Decálogo para la mejora de la docencia online. Propuestas para educar en contextos presenciales discontinuos. Barcelona, Editorial UOC. http://openaccess.uoc.edu/webapps/o2/bitstream/10609/122307/1/9788491807766 _no_venal.pdf

Tucker, C.R.; Wycoff, T. and Green, J.T. (2016). Blended Learning in Action: A Practical Guide Toward Sustainable Change. Corwin Publishers

UNESCO (2020a). Global monitoring of school closures caused by COVID-19. https://en.unesco.org/covid19/educationresponse.

UNESCO (2020b). National Education Responses to COVID-19 Summary report of UNESCO's online survey. https://unesdoc.unesco.org/ark:/48223/pf0000373322

UNESCO (2020c). Framework for reopening schools.

https://unesdoc.unesco.org/ark:/48223/pf0000373348

Análisis de las políticas de enseñanza universitaria en España para tiempos Covid19: la presencialidad adaptada. M. Area. A. Bethencourt, S. Martín y B. San Nicolás.

Página 18 de 19 
RED. Revista de Educación a Distancia. Núm. 65, Vol. 21. Artíc. 3, 08-01-2021.

DOI: https://doi.org/10.6018/red.450461

UNESCO-IESALC (2020). COVID-19 y educación superior: De los efectos inmediatos al día después. Análisis de impactos, respuestas políticas y recomendaciones. Instituto Internacional de la UNESCO para la Educación Superior en América Latina y el Caribe http://www.iesalc.unesco.org/wpcontent/uploads/2020/04/COVID-19-060420-ES-2.pdf

World Bank (2020). The COVID-19 Crisis Response: Supporting tertiary education for continuity, adaptation, and innovation. http://pubdocs.worldbank.org/en/621991586463915490/WB-Tertiary-Ed-and-Covid19-Crisis-for-public-use-April-9.pdf presencialidad adaptada. M. Area. A. Bethencourt, S. Martín y B. San Nicolás. 